\title{
EDITORIAL・ÉDITORIAL
}

\section{Leadership during the COVID-19 crisis and beyond}

The views expressed in this editorial are those of the author and do not necessarily reflect the position of the Canadian Medical Association or its subsidiaries.

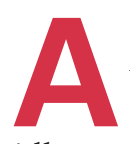
n Internet search for the definition of "leadership" yields 1429 distinct descriptions. This is an interesting result, considering that each of us could rapidly construct a definition we believe to be accurate if asked. This discrepancy is underscored by the relatively recent explosion over the past 15 years or so of courses, documents, lectures and online resources surrounding the concept of leadership. What have become more clear than a unifying definition of leadership, however, are many of the features and concepts that make a skilled leader. More specifically, how does a leader ensure that their group/ team is greater than the sum of its parts? Given the current coronavirus disease 2019 (COVID-19) pandemic and the incredible practice-changing shifts that have occurred as a result, the need for exceptional leadership within our surgical communities has never been greater.

Many of the concepts inherent in strong leadership arise from the arenas of sport, commerce and elite branches of the military. These are the same philosophies that build winning teams across all areas of life. ${ }^{1}$ Although many surgeons have played elite team sports and experienced/used these leadership tools, they are worth repeating during these interesting times. They include 1) hiring people who are smarter than you; 2) listening to your rank and file's ideas; 3) addressing important issues face to face with your team; 4) building environments that feel safe and encourage team members to be honest and authentic and to share vulnerabilities (despite ensuring safe conflict); 5) establishing and maintaining purpose (i.e., the overarching goal beyond the day-to-day practical missions) within each member of the group as well as a greater collective purpose/vision that is clear, repeatable and ever present; 6) constructing environments that allow frequent and close interactions among members (i.e., Allen curve - communication ceases to be effective beyond an $8 \mathrm{~m}$ separation between parties ${ }^{2}$ ); 7) focusing leadership on bar-setting behaviours and outcomes; 8) measuring metrics that really matter; 9) naming, ranking and communicating your group's priorities clearly; 10) encouraging a culture, and constructing a mechanism, that ensures "Kaizen" (i.e., continuous improvement and change); 11) remembering that maximizing purpose is more important than maximizing profit (i.e., Hawthorne effect - emotional reward is more important than material compensation to most team members) ${ }^{3}$; 12) maintaining integrity as a leader (set a visible example and do exactly what you say you will do); and 13) ensuring your organization/team can respond acutely to unexpected events in a manner that navigates uncertainty despite the preceding need to focus on a particular task. ${ }^{3}$ It is also critical to remember that diversity within teams is not only essential in the discussion of equality, but is also absolutely critical to improving a team's ability to solve complex problems and enhance outcomes. This reality has been most eloquently described by Scott Page in his diversity prediction theorem. ${ }^{4}$ Finally, we must be cognisant that leaders are not the same as managers. These terms are frequently misaligned within health care. Leaders are rare; managers are plentiful.

Each of these leadership principles is ideally pursued by our surgical leaders in a time of normalcy. But what happens when an unpredicted event such as a global pandemic occurs? How does this affect traditional concepts within leadership? As numerous world champion boxers have repeated, "everyone has a plan until they get punched in the mouth." To this end, effective leadership within the pandemic should look different. Carlos Pellegrini, past president of the American College of Surgeons, recently stated, "this is not leadership as usual; this is leadership on the edge." ${ }^{5}$ Dr. Pellegrini eloquently outlined a number of updated leadership principles that included 1) be present (being visible is the most important thing a leader can do in a time of crisis to quell anxiety and uncertainty; 2) communicate frequently (outline updates and corresponding actions often); 3) communicate thoroughly (share more rather than less, and connect with the team rather than dictate to the team); 4) communicate truthfully (members will have already collected substantial amounts of information, so err on the side of over-informing and being truthful); and 5) exercise pragmatic optimism (truth anchored in reality). ${ }^{5}$ Effective crisis management includes each of these concepts with an added dose of resilience and patience.

The truth is that despite the negative aspects of the COVID-19 pandemic, it can also be framed as an opportunity. As coach Woodward summarized, "success can be attributed to how a team worked together under pressure, how they understood the importance of teamwork, and how they were willing to do a hundred things just $1 \%$ better." ${ }^{6}$ Times of challenge and stress can also help improve previously poor team performance with the timely addition of exceptional leadership. ${ }^{7}$ The emotional glue of any group or culture remains a common sense of identity and purpose, or in the case of COVID-19, a common enemy. In the end, "great leaders ruthlessly protect their people; encourage connection, collaboration, and collective ownership; and thereby nurture a safe environment of trust, 
respect and family." In many of our careers, this leadership has never been needed more than it is right now. As Charles Darwin reminded us, "it's not the strongest or most intelligent species that survives, but the one most responsive to change." Ensuring connection, collaboration, and communication among our surgical teams in this time of crisis will allow us all to rise out of the COVID-19 era as more focused, efficient and resilient clinicians.

\section{Chad G. Ball, MD, MSc}

Coeditor, Canadian Fournal of Surgery

Competing interests: None declared.

DOI: $10.1503 /$ cjs. 016020

\section{References}

1. Kerr J. Legacy. Constable \& Robinson LTD; 2015.

2. Allen TJ. Managing the flow of technology: technology transfer and the dissemination of technological information within the R\&D organization. Cambridge: MA MIT Press; 1984.

3. Coyle D. The culture code: The secrets of highly successful groups. Penguin Random House; 2017.

4. Page S. The difference: how the power of diversity creates better groups, firms, schools and societies. Princeton, NJ: Princeton University Press; 2007.

5. Hoyt DB. Looking forward. Bulletin of the fournal of the American College of Surgeons. 2020. Available: https://bulletin.facs.org/2020/07/ looking-forward-july-2020/ (accessed 2020 Aug. 10).

6. Woodward C. Winning! Hodder; 2005.

7. Walsh B. The score takes care of itself. Portfolio/Penguin; 2010. 